\title{
Bryophytes of the Parque Estadual da Serra do Brigadeiro, an Atlantic Forest Remnant in Southeastern Brazil ${ }^{1}$
}

\author{
Libia Mayerly Cifuentes-García², 4, 5, (D) Pedro Bond Schwartsburd² and (1) Denilson Fernandes Peralta ${ }^{3}$
}

Submetido: 03.06.20; aceito: 11.09 .20

Como citar: Cifuentes-García, L.M., Schwartsburd, P.B., \& Peralta, D.F. 2020. Bryophytes of the Parque Estadual da Serra do Brigadeiro, an Atlantic Forest Remnant in Southeastern Brazil. Hoehnea 47: e612020. http://dx.doi.org/10.1590/2236-8906-61/2020.

\begin{abstract}
Bryophytes of the Parque Estadual da Serra do Brigadeiro, an Atlantic Forest remnant in Southeastern Brazil). The objective was to present the floristic survey of the bryophytes of the Parque Estadual da Serra do Brigadeiro. It included an evaluation of the species richness, as well as information about the substrate preference of each species and its geographic distributions in Brazil. We found 208 taxa of bryophytes: one hornwort, 88 liverworts and 119 mosses. This richness represents ca. 26\% of the bryoflora of Minas Gerais State and ca. 13\% of that known for Brazil. Eighteen of the bryophytes species are endemic to Brazil, whereas 19 species are recorded for the first time in Minas Gerais. The bryoflora showed preference for tree bark $(30 \%)$ and rock $(23 \%)$ as substrates. Our data show that the Parque Estadual da Serra do Brigadeiro is an important area for the diversity conservation of Brazilian bryophytes and maintenance of endemic species of the country.
\end{abstract}

Keywords: conservation, liverworts, Minas Gerais State, mosses, substrate

RESUMO - (Briófitas do Parque Estadual da Serra do Brigadeiro, um remanescente de Mata Atlântica no Sudeste do Brasil). O objetivo foi apresentar o levantamento florístico das briófitas do Parque Estadual da Serra do Brigadeiro, incluindo uma avaliação da riqueza de espécies, bem como informações sobre a preferência de substrato de cada espécie e suas distribuições geográficas no Brasil. Encontramos 208 táxons de briófitas: um antócero, 88 hepáticas e 119 musgos. Essa riqueza representa 26\% da brioflora do Estado de Minas Gerais e 13\% do Brasil. Dezoito das espécies de briófitas são endêmicas do Brasil, enquanto 19 espécies são registradas pela primeira vez em Minas Gerais. A brioflora mostrou preferência pelo substrato casca de árvore (30\%) e rocha (23\%). Os dados obtidos mostram que o Parque Estadual da Serra do Brigadeiro é uma área importante para a conservação da diversidade de briófitas brasileiras e para a manutenção de espécies endêmicas do país. Palavras-chaves: conservação, hepáticas, Estado de Minas Gerais, musgos, substrato

\section{Introduction}

Bryophytes are nonvascular plants characterized by a life cycle featuring alternating haploid and diploid generations, with the gametophyte as the main life form, which helps and feeds the unbranched, unisporangiate sporophyte (Vanderpoorter \& Goffinet 2009). The monophyly of the three bryophyte groups and the Setaphyta clade of liverworts and mosses as the sister to Traqueophyta has been supported
(Puttick et al. 2018), contrary to the view of three lineages of bryophytes like a paraphyletic group (Goffinet \& Shaw 2009) and hornworts as the sister to vascular plants (Qiu et al. 2006).

These three groups are represented by ca. 18, 000 species worldwide (Goffinet \& Shaw 2009). In Brazil, 1,578 taxa have been recorded (Flora do Brasil 2020, 2020), and the country has the highest rate of endemism (22\%) (Flora do Brasil 2020, 2020).

1. Part of the Masters' Dissertation of the first Author

2. Universidade Federal de Viçosa, Departamento de Biologia Vegetal, Campus Universitário Avenida P.H. Rolfs s/n, 36570-900 Viçosa, MG, Brasil

3. Instituto de Botânica, Núcleo de Pesquisa em Briologia, Avenida Miguel Estefano, 3687, Água Funda, 04301-902 São Paulo, SP, Brasil

4. Fundación Universitaria de Popayán, Sede Claustro San José, Calle 5 No. 8-58, Popayán, Cauca, Colombia

5. Corresponding author: libiacifuentes1986@gmail.com 
Bryophyte representativeness is especially high in the Atlantic forest, which harbors $87 \%$ of all taxa recorded for the country (Flora do Brasil 2020, 2020). This biome is recognized as a biodiversity hotspot with a high percentage of endemic species (Myers et al. 2000, Stehmann et al. 2009), including mosses and liverworts. Unfortunately, it is under intensive process of deforestation and destruction, and only ca. $28 \%$ of the native vegetation cover persists as isolated fragments in different stages of succession, with only $30 \%$ of the total vegetation cover found in protected areas, $9 \%$ of them are strictly protected and $21 \%$ of sustainable use (Rezende et al. 2018). Consequently, this bryoflora could be seriously threatened and many species could disappear without being known to science.

Several floristic surveys of bryophytes in Minas Gerais State were done in the Atlantic Forest, such as Parque Estadual de Ibitipoca (Luizi-Ponzo et al. 2013, Yano \& Luizi-Ponzo 2014, Siviero \& Luizi-Ponzo 2015), Serra Negra (Amorin et al. 2017), and Parque Nacional da Serra do Cipó (Yano \& Peralta 2011a, Sousa \& Câmara 2015). However, due the great size of the state, there are still large gaps in the knowledge of the brioflora of Minas Gerais State (Amorin 2013).

The Parque Estadual da Serra do Brigadeiro (PESB) is located in one of the areas of the Atlantic Forest that still comprises large tracts of continuous forest (Plano de Manejo PESB- Parque Estadual da Serra do Brigadeiro 2007). The Park has a variety of geographical, climatic and phytophysiological conditions, and, consequently, it harbors a high quality and quantity of microhabitats, with variation in the availability of nutrients (Benites et al. 2001), a key factor in the diversity patterns of bryophytes (Pócs 1982, Newmaster et al. 2005). For these reasons, the PESB harbors a diverse community of bryophytes with high numbers of endemic species, many of which have not been previously recorded for the state of Minas Gerais.

Despite its relevance, the bryoflora of the PESB is still poorly known. There are only three papers citing bryophytes collected in the PESB: Crum (1994), describing a new species, Sphagnum leonii, Leoni \& Tinte (2004) listing 52 species that occur in the area, and Castro et al. (2011) listing the herbarium specimens.

In this paper, we present a detailed floristic survey of the bryophytes of the PESB, including an assessment of species richness, as well as information on substrate preference of each species, and its geographical distributions at the national level according to Flora do Brasil 2020 (2020).

\section{Materials and methods}

Study site - The Parque Estadual da Serra do Brigadeiro (PESB) is a protected area under state administration, located among the municipalities of Ervália, Fervedouro, Sericita, Araponga, Miradouro, Pedra Bonita, Muriaé and Divino, in the State Minas Gerais, Brazil. The Park covers an area of 14,985 ha between the geographical coordinates $20^{\circ} 33^{\prime}$ to $21^{\circ} 00^{\prime} \mathrm{S}$ and $42^{\circ} 40^{\prime}$ to $40^{\circ} 20^{\prime} \mathrm{W}$ (figure 1, map was generated using ArcGIS ${ }^{\circledR}$ software). The vegetation in the Park is composed of secondary fragments inserted in the area of Atlantic Forest biome (Caiafa \& Silva 2005) and has two types of vegetal formations, according with IBGE (2012): (1) semideciduous forest from the altomontana formation and (2) high-altitude fields (campos de altitude).

Floristic surveys - Specimens were collected during four field expeditions between May 2017 and April 2018, along eight preexistent trails located in the northern, central and southern areas of the Park (approximate coordinates plotted in figure 1). Collecting effort was intended to cover the range of habitats and microhabitats (substrate type) available for bryophytes, like soil, trunks, lianas, shrubs, rocks, in both semideciduous forest and high-altitude fields, between 1000 to $1540 \mathrm{~m}$ of elevation, following Frahm's recommendations (2003). Specimens were deposited at VIC herbarium, with duplicates in SP (Acronyms follow Index Herbariorum, Thiers, 2020).

Bryophyte identification - For the specimen identification we used keys and specialized literature for to each family (Yano \& Peralta 2011a, Frahm 1991, Sharp et al. 1994, Yano \& Carvalho 1995, Gradstein, Churchill, et al. 2001, Gradstein \& Costa 2003, Bordin \& Yano 2013).

Our classification follows Söderström et al. (2016) for Anthocerotophyta and Marchantiophyta and Goffinet et al. (2009) for Bryophyta. The taxa are listed in alphabetical order by families, genera, and species, indicating endemic species and those recorded for the first time for Minas Gerais State. Distribution data was obtained from Flora do Brasil 2020 (2020), as well as from Santos et al. (2017), Carmo \& Peralta (2016), Costa \& Peralta (2015), Machado et al. (2015), Yano \& Luizi-Ponzo (2014), Yano \& Peralta (2011a), and Gradstein \& Costa (2003).

Species classification according to substrate types follows Robbins (1952): (1) corticolous, species that grow on living trunks and branches; (2) epiphyllous, those that grow on leaves; (3) epixylic, on dead or decaying trunk; (4) rupicolous, on stones; and (5) terrestrial, on the ground.

\section{Results}

We collected and analyzed 514 specimens, corresponding to 208 species of bryophytes: one hornwort, 88 liverworts (20 families, 41 genera) and 119 mosses (32 families, 63 genera), (table 1). These taxa comprise 208 species, three varieties and one subspecies.

The best represented liverworts families were Lejeuneaceae, with 35 species (36\%), Plagiochilaceae, with $10(11 \%)$, and Lepidoziaceae, with six $(6 \%)$, whereas the best represented moss families were Leucobryaceae, with 16 species (13\%), Sematophyllaceae, with $12(10 \%)$, and Pilotrichaceae, with nine (7\%). The most representative genera in liverworts were Plagiochila and Lejeunea, with 10 species each; in mosses, the most speciose genera were Campylopus, with 10 species, and Fissidens, with nine, (table 1). 
Table 1. List of bryophytes found in the Parque Estadual da Serra do Brigadeiro, Minas Gerais State, Brazil, with their substrate. Voucher information corresponds to the collecting series of Libia Mayerly Cifuentes (LMC). *: New record for Minas Gerais State. **: Endemic species. ***: New record for Minas Gerais State and Endemic species.

\begin{tabular}{lll}
\hline Taxa & Substrato & Vouch \\
\hline Anthocerotophyta & & \\
Anthocerotaceae & & 659 \\
$\quad$ Anthoceros punctatus L. & Soil & \\
Bryophyta & & 350 \\
Bartramiaceae & & 351 \\
Breutelia tomentosa (Sw. ex Brid.) A. Jaeger & Soil & 479 \\
Leiomela bartramioides (Hook.) Paris & Rock/Tree bark & 514 \\
Philonotis sphaerocarpa (Hedw.) Brid. & Rock/Soil & Rock \\
Philonotis uncinata (Schwägr.) Brid. & &
\end{tabular}

Brachytheciaceae

Rhynchostegium serrulatum (Hedw.) A. Jaeger

Soil/Tree bark 659

Squamidium brasiliense Broth.

Rock/Soil

Zelometeorium patens (Hook.) Manuel

Rock/Tree bark

Zelometeorium patulum (Hedw.) Manuel

Rock/Tree bark

Bryaceae

**Brachymenium hornschuchianum Mart.

Tree bark

Brachymenium patulum (Müll. Hal.) Schimp. ex Besch.

Tree bark

Bryum argenteum Broth.

Rock

Bryum cellulare Hook.

Rock

**Bryum subapiculatum

Soil

477

Rhodobryum subverticillatum Broth.

Rock

Rosulabryum canariense (Brid.) Ochyra

Soil

495

Rosulabryum densifolium (Brid.) Ochyra

Rock/Soil

Calymperaceae

Octoblepharum albidum Hedw.

Rock

**Syrrhopodon brasiliensis W. D. Reese

Tree bark

Syrrhopodon gaudichaudii Mont.

Tree bark

536

Syrrhopodon incompletus Schwägr.

Decayed wood

690

Syrrhopodon leprieuri Mont.

Rock

446

Syrrhopodon prolifer Schwägr.

Decayed wood/Tree bark/Rock/Soil

Syrrhopodon tortilis Hampe

Rock

Cryphaeacea

Schoenobryum concavifolium (Griff.) Gangulee

Tree bark

Daltoniaceae

Calyptrochaeta setigera (Mitt.) W. R. Buck

Tree bark

Dicranaceae

Holomitrium arboreum Mitt.

Soil

Holomitrium crispulum Mart.

Rock 
Table 1 (continuation)

Taxa

Entodon argyreus (Besch.) Besch.

Rock

Fissidentaceae

Fissidens asplenioides Hedw.

Rock

*Fissidens dissitifolius Sull.

Tree bark

716

Fissidens elegans Brid.

Rock

580

Fissidens hornschuchii Mont.

Rock/Tree bark

725

Fissidens pellucidus Hornsch

Tree bark

760

Fissidens saprophilus Broth.

Tree bark

539

Fissidens scariosus Mitt.

Soil

507

Fissidens zollingeri Mont.

Rock

756

Hookeriaceae

*Crossomitrium epiphyllum (Mitt.) Müll. Hal.

Tree bark

646

*Crossomitrium saprophilum Broth.

Rock

Hypnaceae

Chryso-hypnum diminutivum (Hampe) W. R. Buck

Rock/Soil/Tree bark

Chryso-hypnum elegantulum (Hook.) Hampe

Rock

680

Mittenothamnium reptans (Hedw.) Cardot

Tree bark

Hypopterygiaceae

Hypopterygium tamariscinum (Hedw.) Brid.

Tree bark

*Lopidium concinnum (Hook.) Wilson

Rock/Tree bark

600

Lembophyllaceae

Orthostichella pachygastrella (Müll. Hal. ex Ångstr.) B. H.

Allen \& Magill

Tree bark

Pilotrichella flexilis (Hedw.) Ångström

Tree bark

Leucobryaceae

Campylopus aemulans (Hampe) A. Jaeger

Rock

Campylopus arctocarpus (Hornsch.) Mitt.

Soil

Campylopus filifolius (Hornsch.) Mitt. var. humile

Soil/Tree bark

Campylopus filifolius (Hornsch.) Mitt. var. filifolius

Tree bark

522

Campylopus gastroalaris (Müll.Hal.) Paris

Tree bark

540

Campylopus julaceus A. Jaeger

Rock

497

Campylopus lamellinervis (Müll.Hal.) Mitt.

Soil

482

Campylopus pilifer Brid.

Rock

570

Campylopus savannarum (Müll. Hal.) Mitt.

Tree bark

516

**Leucobryum clavatum Hampe

Soil/Tree bark

372

Leucobryum crispum Müll. Hal.

Soil

559

Leucobryum laevifolium Broth.

Rock

576

Pilopogon guadalupensis (Brid.) J. -P. Frahm

Rock

Meteoriaceae 
Table 1 (continuation)

\begin{tabular}{llc}
\hline Taxa & Substrato & Voucher \\
\hline Papillaria callochlorosa Müll. Hal. & Tree bark & 647 \\
Mniaceae & & \\
Plagiomnium rhynchophorum (Hook.) T.J.Kop. & Rock/Soil /Tree bark & 353
\end{tabular}

Neckeraceae

Neckera ehrenbergii Müll. Hal.

Tree bark $\quad 588$

Neckeropsis disticha (Hedw.) Kindb.

Tree bark 642

*Porotrichodendron superbum (Taylor) Broth.

Rock

Porotrichum lancifrons (Hampe) Mitt.

Rock

Porotrichum longirostre (Hook.) Mitt.

Rock/Tree bark

Orthodontiaceae

Hymenodon aeruginosus (Hook. f. \& Wilson) Müll. Hal.

Rock/Tree bark

Orthotrichaceae

*Groutiella apiculata (Hook.) H. A. Crum \& Steere

Tree bark

Groutiella tomentosa (Hornsch.) Wijk \& Margad.

Macrocoma tenuis subsp. sullivantii (Müll.Hal.) Vitt

Tree bark

Macromitrium microstomum (Hook. \& Grev.) Schwägr.

Tree bark

Tree bark

Macromitrium punctatum (Hook. \& Grev.) Brid.

Tree bark

Macromitrium richardii Schwägr.

Tree bark

Macromitrium podocarpi Müll. Hal.

Tree bark

Schlotheimia appressifolia (Hornsch.) Wijk \& Margad.

Tree bark

Schlotheimia jamesonii (Arn.) Brid.

Tree bark

Phyllogoniaceae

Phyllogonium viride Brid.

Rock/Tree bark

Pilotrichaceae

Cyclodictyon albicans (Hedw.) Kuntze

Rock

Cyclodictyon limbatum (Hampe) Kuntze

Tree bark

***Lepidopilum caudicaule (Müll.Hal.) Broth.

Rock/Tree bark

Lepidopilum muelleri (Hampe) Mitt.

Rock/Tree bark

762

Lepidopilum scabrisetum (Schwägr.) Steere

Rock

758

Thamniopsis incurva (Hornsch.) W. R. Buck

Soil/Decayed wood

561

Thamniopsis langsdorffii (Hook.) W. R. Buck

Rock/Tree bark

724

*Trachyxiphium aduncum (Mitt.) W. R. Buck

Soil

Trachyxiphium saxicola (R. S. Williams) Vaz-Imbassahy \& Costa

Rock/Tree bark

Polytrichaceae

Pogonatum campylocarpum (Müll. Hal.) Mitt.

Soil

**Polytrichum angustifolium Mitt.

Polytrichum commune L. ex Hedw.

391

Polytrichum juniperinum Willd. ex Hedw.

Rock

356

Pottiaceae 
Table 1 (continuation)

\begin{tabular}{llc}
\hline Taxa & Substrato & Voucher \\
\hline Hyophila involuta (Hook.) A. Jaeger & Rock & 478 \\
Tortella humilis (Hedw.) Jenn. & Rock & 480
\end{tabular}

Prionodontaceae

Prionodon densus (Hedw.) Müll. Hal.

Tree bark

Pylaisiadelphaceae

Isopterygium subbrevisetum (Hampe) Broth.

Tree bark

708

Isopterygium tenerum (Sw.) Mitt.

Tree bark

706

*Pylaisiadelpha tenuirostris (Bruch \& Schimp.) W. R. Buck

Decayed wood

685

Wijkia flagellifera (Broth.) H. A. Crum

Tree bark

Racopilaceae

Racopilum tomentosum (Hedw.) Brid.

Rock/Tree bark

Rhacocarpaceae

**Rhacocarpus inermis (Müll. Hal.) Lindb. var. inermis

Rock

Rhizogoniaceae

Pyrrhobryum spiniforme (Hedw.) Mitt.

Decayed wood/Soil/Tree bark

Sematophyllaceae

**Aptychopsis estrellae (Hornsch.) Ångström

Tree bark

**Aptychopsis pyrrophylla (Müll.Hal.) Wijk \& Margad.

Rock/Decayed wood

**Aptychopsis subpungifolia (Broth.) Broth.

Tree bark

Brittonodoxa subpinnata (Brid.) W. R. Buck, P. E. A. S. Câmara \& Carv.-Silva

Rock/Tree bark

Microcalpe subsimplex (Hedw.) W. R. Buck

Rock/Soil/Tree bark

Sematophyllum pectinatum (Herzog) Schäf.-Verw.

Rock/Tree bark

Sematophyllum subdepressum (Hampe) Broth.

Rock/Tree bark

Sematophyllum swartzii (Schwägr.) W. H. Welch \& H. A. Crum

Soil/Tree bark

Vitalia cuspidifera (Mitt.) P. E. A. S. Câmara et al.

Rock

732

Vitalia galipensis (Müll. Hal.) P. E. A. S. Câmara et al.

Decayed wood

Sphagnaceae

**Sphagnum gracilescens Müll. Hal.

Rock

Sphagnum perichaetiale Hampe

Rock

655

Sphagnum sparsum Hampe

Sphagnum subsecundum Nees

Rock

700

Stereophyllaceae

Eulacophyllum cultelliforme (Sull.) W. R. Buck \& Ireland

Tree bark

Thuidiaceae

Pelekium schistocalyx (Müll.Hal.) A. Touw

Soil/Tree bark

**Thuidium bifidum A. Soares \& P. E. A. S. Câmara

Soil

671

Thuidium delicatulum (Hedw.) Schimp.

Soil/Tree bark

550

Thuidium tomentosum Schimp.

Rock/Soil/Tree bark

Marchantiophyta 
Table 1 (continuation)

\begin{tabular}{|c|c|c|}
\hline Taxa & Substrato & Voucher \\
\hline \multicolumn{3}{|l|}{ Adelanthaceae } \\
\hline Syzygiella rubricaulis (Nees) Grolle & Rock/Tree bark & 546 \\
\hline \multicolumn{3}{|l|}{ Aneuraceae } \\
\hline Aneura pinguis (L.) Dumort. & Decayed wood & 565 \\
\hline Riccardia chamedryfolia (With.) Grolle & Tree bark & 501 \\
\hline Riccardia digitiloba (Spruce ex Steph.) Pagán & Decayed wood & 596 \\
\hline **Riccardia emarginata (Steph.) Hell & Soil & 568 \\
\hline Riccardia metzgeriiformis (Steph.) R. M. Schust. & Rock/Soil & 658 \\
\hline **Riccardia regnellii (Aongström.) Hell & Rock & 465 \\
\hline \multicolumn{3}{|l|}{ Balantiopsidaceae } \\
\hline Neesioscyphus bicuspidatus (Steph.) Grolle & Soil & 669 \\
\hline Neesioscyphus argillaceus (Nees) Grolle & Soil & 676 \\
\hline \multicolumn{3}{|l|}{ Calypogeiaceae } \\
\hline Calypogeia peruviana Nees \& Mont. & Soil & 568 \\
\hline \multicolumn{3}{|l|}{ Cephaloziaceae } \\
\hline $\begin{array}{l}\text { **Fuscocephaloziopsis crassifolia (Lindenb. \& Gottsche) Váňa } \\
\text { \& L. Söderstr }\end{array}$ & Tree bark & 558 \\
\hline Odontoschisma variabile (Lindenb. \& Gottsche) Trevis. & Tree bark & 558 \\
\hline${ }^{*}$ Cephaloziopsis intertexta (Gottsche) R. M. Schust. & Rock & 581 \\
\hline \multicolumn{3}{|l|}{ Dumortieraceae } \\
\hline Dumortiera hirsuta (Sw.) Nees & Rock/Soil & 420 \\
\hline \multicolumn{3}{|l|}{ Frullaniaceae } \\
\hline Frullania brasiliensis Raddi & Tree bark & 360 \\
\hline Frullania caulisequa (Nees) Nees & Tree bark & 546 \\
\hline Frullania ericoides (Nees) Mont. & Tree bark & 462 \\
\hline Frullania riojaneirensis (Raddi) Spruce & Tree bark & 456 \\
\hline \multicolumn{3}{|l|}{ Jungermanniaceae } \\
\hline Jungermannia amoena Lindenb. \& Gottsche & Soil & 669 \\
\hline \multicolumn{3}{|l|}{ Lejeuneaceae } \\
\hline Acrolejeunea emergens (Mitt.) Steph. & Tree bark & 473 \\
\hline Anoplolejeunea conferta (C. F. W. Meissn.) A. Evans & Tree bark & 536 \\
\hline Bryopteris filicina (Sw.) Nees & Rock/Tree bark & 378 \\
\hline Cheilolejeunea acutangula (Nees) Grolle & Tree bark & 574 \\
\hline Cheilolejeunea clausa (Nees \& Mont.) R. M. Schust. & Rock & 710 \\
\hline Cheilolejeunea comans (Spruce) R. M. Schust. & Tree bark & 699 \\
\hline Cheilolejeunea filiformis (Sw.) W. Ye, R. L. Zhu \& Gradst. & Soil/Tree bark & 464 \\
\hline Cheilolejeunea rigidula (Nees ex Mont.) R.M. Schust. & Rock & 709 \\
\hline Drepanolejeunea anoplantha (Spruce) Steph. & Leaves & 463 \\
\hline Drepanolejeunea granatensis (J. B. Jack \& Steph.) Bischl. & Leaves & 537 \\
\hline Frullanoides tristis (Steph.) van Slageren & Tree bark & 457 \\
\hline
\end{tabular}


Table 1 (continuation)

\begin{tabular}{|c|c|c|}
\hline Taxa & Substrato & Voucher \\
\hline *Lejeunea bermudiana (A. Evans) R.M.Schust. & Leaves & 438 \\
\hline Lejeunea caulicalyx (Steph.) E. Reiner \& Goda & Leaves & 723 \\
\hline **Lejeunea cristulata (Steph.) E. Reiner \& Goda & Rock & 715 \\
\hline Lejeunea deplanata Nees & Tree bark & 276 \\
\hline Lejeunea flava (Sw.) Nees & Decayed wood & 736 \\
\hline Lejeunea laetevirens Nees \& Mont. & Rock/Tree bark & 411 \\
\hline **Lejeunea oligoclada Spruce & Leaves & 438 \\
\hline Lejeunea setiloba Spruce & Leaves/Tree bark & 531 \\
\hline Lopholejeunea nigricans (Lindenb.) Schiffn. & Tree bark & 711 \\
\hline Marchesinia brachiata (Sw.) Schiffn. & Tree bark & 492 \\
\hline Neurolejeunea breutelii (Gottsche) A. Evans & Rock & 508 \\
\hline *Prionolejeunea aemula (Gottsche) A. Evans & Leaves & 416 \\
\hline *Prionolejeunea denticulata (Weber) Schiffn. & Leaves & 411 \\
\hline *Prionolejeunea mucronata (Sande Lac.) Steph. & Leaves & 409 \\
\hline Schiffneriolejeunea polycarpa (Nees) Gradst. & Tree bark & 483 \\
\hline *Lejeunea asthenica (Spruce) & Tree bark & 404 \\
\hline Lejeunea pterigonia (Lehm. \& Lindenb.) Mont. & Rock/Tree bark & 726 \\
\hline *Lejeunea serpillifolioides (Raddi) Gradst. & Rock & 363 \\
\hline \multicolumn{3}{|l|}{ Lepidoziaceae } \\
\hline Bazzania stolonifera (Sw.) Trevis. & Leaves & 689 \\
\hline Kurzia brasiliensis (Steph.) Grolle & Rock & 605 \\
\hline Lepidozia cupressina (Sw.) Lindenb. & Tree bark & 606 \\
\hline Lepidozia inaequalis (Lehm. \& Lindenb.) Lehm. \& Lindenb. & Tree bark & 537 \\
\hline Telaranea diacantha (Mont.) Engel \& Merr. & Decayed wood/ Rock/ Soil/Tree bark & 554 \\
\hline Telaranea nematodes (Gottsche ex Austin) & Rock & 465 \\
\hline \multicolumn{3}{|l|}{ Lophocoleaceae } \\
\hline Cryptolophocolea martiana (Nees) & Decayed wood/ Rock/ Soil/Tree bark & 404 \\
\hline Lophocolea bidentata (L.) Dumort. & Decayed wood/ Rock/ Soil/Tree bark & 501 \\
\hline Lophocolea muricata (Lehm.) Nees & Decayed wood/Tree bark & 537 \\
\hline \multicolumn{3}{|l|}{ Marchantiaceae } \\
\hline Marchantia chenopoda $\mathrm{L}$. & Rock & 419 \\
\hline Marchantia papillata Raddi & Rock/Soil & 494 \\
\hline \multicolumn{3}{|l|}{ Metzgeriaceae } \\
\hline Metzgeria albinea Spruce & Rock/Tree bark & 369 \\
\hline Metzgeria conjugata Lindb. & Rock/Tree bark & 750 \\
\hline Metzgeria fruticola Spruce & Rock & 739 \\
\hline Metzgeria furcata (L.) Dumort. & Tree bark & 644 \\
\hline *Metzgeria hegewaldii Kuwah. & Tree bark & 458 \\
\hline \multicolumn{3}{|l|}{ Monocleaceae } \\
\hline Monoclea gottschei Lindb. & Rock & 468 \\
\hline
\end{tabular}


Table 1 (continuation)

\begin{tabular}{|c|c|c|}
\hline Taxa & Substrato & Voucher \\
\hline \multicolumn{3}{|l|}{ Noterocladaceae } \\
\hline Noteroclada confluens Taylor ex Hook. \& Wilson & Soil & 669 \\
\hline \multicolumn{3}{|l|}{ Pallaviciniaceae } \\
\hline Pallavicinia lyellii (Hook.) S. F. Gray & Soil & 672 \\
\hline Symphyogyna aspera Steph. & Rock/Soil & 469 \\
\hline Symphyogyna brasiliensis (Nees) Nees \& Mont. & Rock/Soil Rock & 703 \\
\hline Symphyogyna podophylla (Thunb.) Mont. \& Nees & Rock/Soil & 421 \\
\hline \multicolumn{3}{|l|}{ Plagiochilaceae } \\
\hline Plagiochila adianthoides (Sw.) Lindenb. & Rock & 426 \\
\hline Plagiochila bifaria (Sw.) Lindenb. & Decayed wood/Tree bark & 532 \\
\hline Plagiochila corrugata (Nees) Nees \& Mont. & Rock/Tree bark & 764 \\
\hline Plagiochila martiana (Nees) Lindenb. & Tree bark & 518 \\
\hline Plagiochila patentissima Lindenb. & Rock/Tree bark & 733 \\
\hline Plagiochila patula (Sw.) Lindenb. & Tree bark & 601 \\
\hline Plagiochila rutilans Lindenb. & Rock/Tree bark & 500 \\
\hline Plagiochila simplex (Sw.) Lindenb. & Rock & 412 \\
\hline Plagiochila subplana Lindenb. & Rock & 725 \\
\hline \multicolumn{3}{|l|}{ Porellaceae } \\
\hline Porella brasiliensis (Raddi) Schiffn. & Rock/Tree bark & 492 \\
\hline Porella reflexa (Lehm. \& Lindenb.) Trevis. & Rock & 712 \\
\hline Porella swartziana (Weber) Trevis. & Rock/Tree bark & 430 \\
\hline \multicolumn{3}{|l|}{ Radulaceae } \\
\hline Radula mammosa Spruce & Tree bark & 536 \\
\hline Radula nudicaulis Steph. & Rock/Tree bark & 444 \\
\hline Radula recubans Taylor & Rock/Tree bark & 583 \\
\hline Radula sinuata Gottsche ex Steph. & Tree bark & 539 \\
\hline Radula javanica Gottsche & Rock & 721 \\
\hline \multicolumn{3}{|l|}{ Trichocoleaceae } \\
\hline *Leiomitria flaccida (Spruce) J. B. Jack \& Steph. & Tree bark & 367 \\
\hline
\end{tabular}

Regarding substrate colonization, $70 \%$ of the species were found in only one type of substrate: tree bark (63 taxa), rocks (49), soil (20), decayed wood (six), and live leaves (nine). The remaining species were found in two to four types of substrates.

Eighteen of the bryophyte species are endemic to Brazil, including 13 species of mosses and five liverworts, whereas 19 species $(10 \%)$ are recorded for the first time in the Minas Gerais State (Flora do Brasil 2020 (2020), Carmo \& Peralta 2016, Costa \& Peralta 2015, Machado et al. 2015, Yano \& Luizi-Ponzo 2014, Yano \& Peralta 2011a, Gradstein \& Costa 2003) (table 1).

\section{Discussion}

Bryophyte species richness and Brazilian distribution - The high bryophyte species diversity in the mountain ecosystems of the Neotropics has been related to the large variety of microhabitats and conditions of constant humidity that facilitate the co-occurrence of numerous species (Frahm 2003, Gradstein et al. 2008). The bryophytes found in this survey of the PESB correspond to ca. $26 \%$ of the species estimated for the State of Minas Gerais and $13 \%$ of those known for Brazil (Flora do Brasil 2020, 2020). In our list, we added 189 species to those previously recorded by 


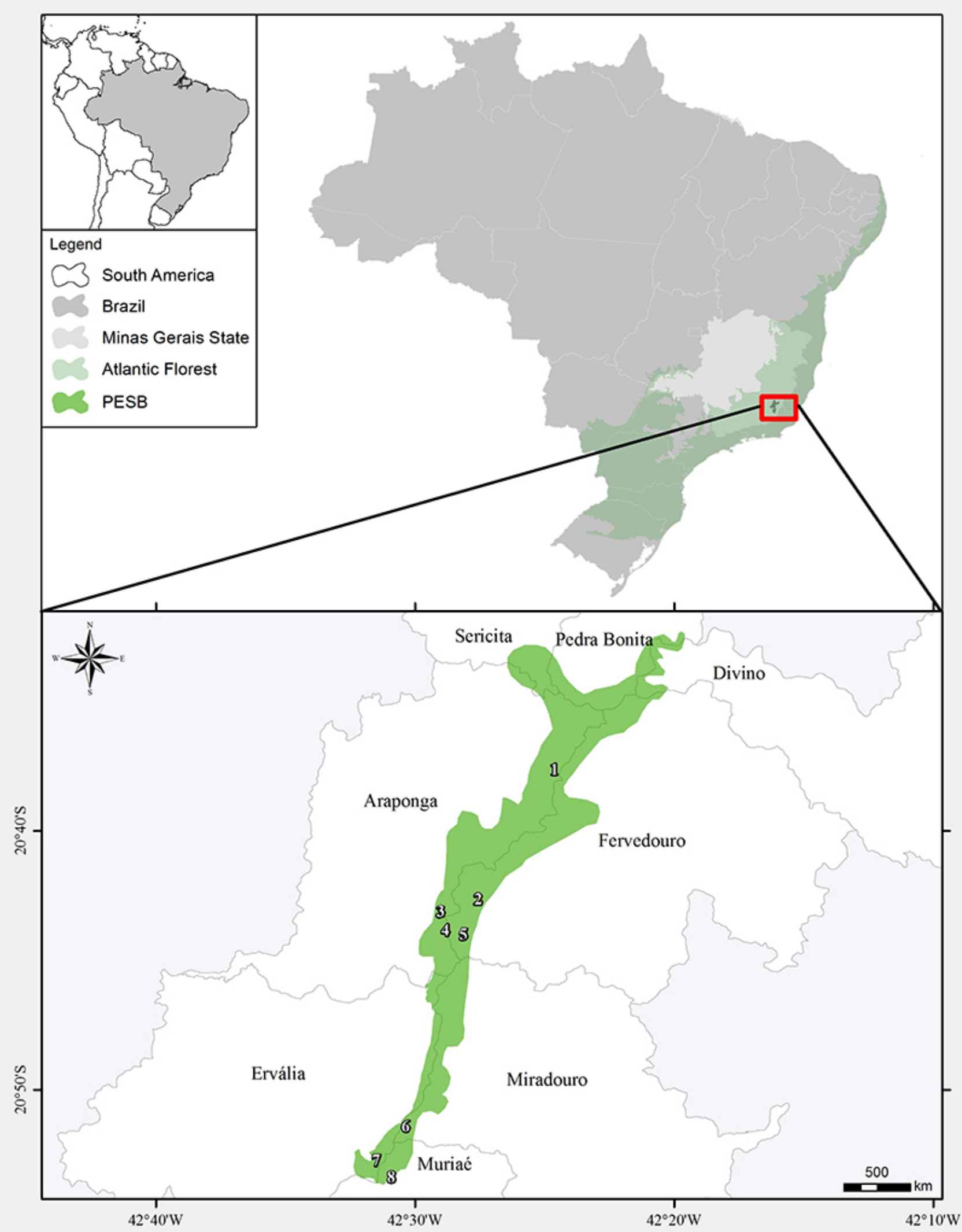

Figure 1. Location of Atlantic Forest domain in Brazil and sampling areas for bryophytes in the Parque Estadual da Serra do Brigadeiro in Minas Gerais State, Brazil. 1. Trilha do Laje do Ouro. 2. Trilha do Carvão. 3. Trilha do Pico do Grama. 4. Trilha da Lajinha. 5. Trilha da Pedra do Pato. 6. Trilha do Cruzeiro do Careço. 7. Trilha do Itajurú. 8. Trilha do Avião. 
Leoni \& Tinte (2004). The difference in species number is large, and the number of families and genera recorded are different, with 53 families and 105 genera versus 26 families and 40 genera listed in Leoni \& Tinte (2004). In their list, these authors included the endemic species Sphagnum leoni, whose type locality is the PESB, and included also six other species that are doubtful records for Brazil.

Our results indicate a high diversity of bryophytes in the PESB. Yano \& Peralta (2011a) found 237 species in the Parque Estadual da Serra do Cipó, Luizi-Ponzo et al. (2013) found 209 taxa in Parque Estadual de Ibitipoca, Amorim et al. (2017) recognized 92 species of the Serra Negra, and Carmo \& Peralta (2016) recorded 289 species in Parque Nacional da Serra da Canastra.

Mosses presented a greater number of species than liverworts. This fact is common in high mountain ecosystems rather than in lowlands (Gradstein et al. 2001b) and this is consistent with results provided in other studies in high altitude areas (Yano \& Peralta 2011a, 2011b, Luizi-Ponzo et al. 2013, Carmo \& Peralta 2016). The predominance of mosses is explained by Costa et al. (2015) as a result of their life forms and the morphology of their gametophyte, which increases the ability to colonize open environments, such as rock outcrops, exposed soils, and banks; these microhabitats are abundant in the PESB, due to the wide altitudinal range of the area.

Lejeuneaceae was the best represented family in terms of species number, with $36 \%$ of the species. This comes as no surprise, as Lejeuneaceae is the largest family of liverworts in Brazil and in the tropics (Gradstein et al. 2001b, Gradstein \& Costa 2003). Another well-represented family was Leucobryaceae (11\%), a group reported by Yano \& Peralta (2011a), Siviero \& Luizi-Ponzo (2015) and Carmo \& Peralta (2016) as having the greatest richness in high altitude areas in Minas Gerais State. The predominance of Leucobryaceae has been attributed to the acrocarpous growth habit of their gametophyte. Furthermore, they have hyalocysts cells in the leaves and alar cells at the basal angles of the leaves (Campylopus), which can store large amounts of water and thus can thrive in more exposed areas (Frahm 2003), a common habitat in the PESB.

On the other hand, $10 \%$ of the species found in the Park are endemic to Brazil (Flora do Brasil 2020 (2020), Costa $\&$ Peralta 2015). Some endemic species in the PESB are Aptychopsis estrellae, Sphagnum gracilescens, Riccardia regnellii, Rhacocarpus inermis var. inermis, Polytrichum angustifolium and Bryum subapiculatum (figure 2) (Flora do Brasil 2020 (2020), Costa \& Peralta 2015, Machado et al. 2015, Yano \& Luizi-Ponzo 2014, Gradstein \& Costa 2003). For that reason, we assume that the PESB offers microhabitats of high quality and quantity, with moisture and nutrients available for these species; these variables have been pointed as determinants of diversity patterns in this group (Pócs 1982, Newmaster et al. 2005).
Substrate colonization - In the PEBS, most bryophytes showed substrate type preferences, with a greater number of species found in tree bark (63 taxa). This predominance could be related to heterogeneous conditions, such as the varied sizes of the phorophytes and the physical characteristics of bark -with more available colonization niches- (Bates 1992, Frahm 2003, Batista et al. 2018), combined with forest conditions of temperature, luminosity, and humidity (Batista \& Santos 2016). In works done in the Atlantic Forest, Germano \& Pôrto (2005), Sousa \& Câmara (2015) and Siviero \& Luizi-Ponzo (2015) also observed a greater number of corticicolous species and they also point to the reasons stated above.

The preference for substrate types has been attributed to the low humidity and to the need of occupying specialized microhabitats, to take advantage of their particular conditions (Batista et al. 2018). The annual average rainfall in the PEBS $(1,300 \mathrm{~mm} /$ year $)$ and it is characterized by a rainy period, during the months of November to March, which is the warmest, and by a dry period, from April to October (Plano de Manejo PESB- Parque Estadual da Serra do Brigadeiro 2007), so the preference for substrate types in bryophytes species of the PESB could be an important strategy for this climate conditions.

The frequency of species on rocky substrates (49 taxa) could be due to the abundant naked surfaces, mainly in the rocky outcrops and streams. In the Atlantic Forest numerous taxa grew on rocks and few species occurred on soil, probably as a result of the existence of a litter on the forest ground that makes difficult growth of the bryophytes (Santos \& Costa 2008). In addition, a lower percentage of species growing in rocks and soil in the Atlantic Forest of northeastern Brazil was explained due to a minor heterogeneity in these substrates in contrast with tree bark (Batista et al. 2018).

On the other hand, epiphyllous species were scarce at our study site (nine taxa), and were represented only by liverworts: eight species of Lejeuneaceae and Bazzania stolonifera (table 1). The greatest representativeness of Lejeuneaceae in this substrate is a common condition in tropical forests (Pócs 1982, Siviero \& Luizi-Ponzo 2015), because content the most specialized epiphyllous species among all the bryophytes, and these have vegetative and reproductive structures for water-storing and anchoring on the leaves (Pócs 1982). Another important attribute of the epiphyllous bryophytes is the heterogeneous occurrence pattern in the forest environment - they are strategically positioned in locations with specific microclimatic conditions (Alvarenga \& Porto 2007). So, low occurrence of epiphyllous species in a general inventory like this one is highly probable, because we mainly sample the easily accessible places (near to pre-existent trails) and these are more readily impacted by different anthropic pressures, such as deforestation, wood extraction, unsustainable tourism (Plano de Manejo PESB - Parque Estadual da Serra do Brigadeiro 2007). 

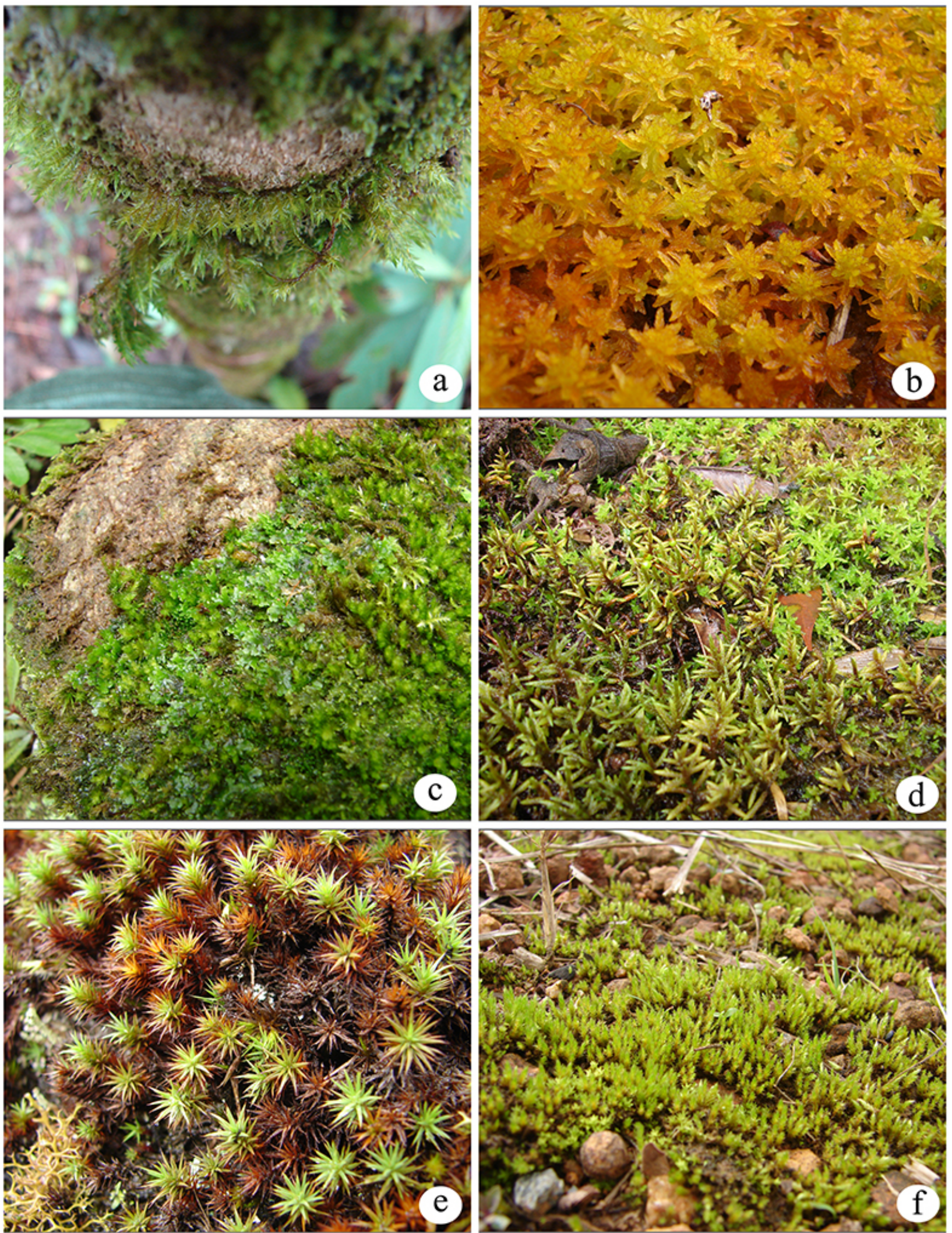

Figure 2. Some endemic species of Brazil found in the Parque Estadual da Serra do Brigadeiro in Minas Gerais State, Brazil. Aptychopsis estrellae (Hornsch.) Ångström, Sphagnum gracilescens Müll. Hal., Riccardia regnellii (Aongström.) Hell, Rhacocarpus inermis (Müll. Hal.) Lindb. var. inermis, Polytrichum angustifolium Mitt. and Bryum subapiculatum Hampe. 
In the fragmented Brazilian Atlantic Forest, the opening of the canopy was considered a cause of reduction and local extinction of epiphyllous bryophyte communities, as it can alter humidity, temperature, and light conditions in the lower forest (Alvarenga \& Porto 2007). Epiphyllous dependence on the microclimate conditions created by vegetation, and consequent susceptibility to environmental disturbances has been repeatedly pointed out (Gradstein 1997, Costa 1999, Alvarenga \& Porto 2007, Alvarenga et al. 2009).

Then, it is advisable to do studies focused on the specific habitats that these plants occupy, such as humid and shady places within the forest (Gradstein et al. 2001), because epiphyllous are good indicators of the state of conservation of the area.

\section{Conclusions}

The data obtained in this study show that the Parque Estadual da Serra do Brigadeiro (PESB) is an important area for conservation of the diversity of Brazilian bryophytes and for the conservation and maintenance of species that are endemics, rare or have a moderate distribution in Brazil.

Our floristic survey of the bryophytes in the PESB contributes to the knowledge of the floristic diversity of the Park with the verification of their distribution, endemism and substrate types. It also contributes with the enrichment of the collection of bryophytes in the VIC Herbarium, where the samples are available for future studies on other aspects of Brazilian bryophytes.

\section{Acknowledgements}

We thank the CAPES (Coordenação de Aperfeiçoamento de Pessoal de Nivel Superior), for providing scholarships by the first Author and Instituto Estadual de Florestas de Minas Gerais (IEF), by permitted sampling. We thank the director of the Parque Estadual da Serra do Brigadeiro, Mr. José Roberto Mendes de Oliveira, and other Park staff, for logistics, transport and assistance during sampling. Thanks so much to Park staff Francisco Javier Oliveira Ramos and Rosmeri Menendes de Paula, who assisted in the collection and transport. We greatly appreciate the help of Nayara Smith and Julio Lanazca Vargas during field work, the contributions of Rodrigo Bernal to the manuscript and Michael Alejandro Castro Bonilla by the map elaboration.

\section{Literature cited}

Alvarenga, L.D.P. \& Pôrto, K.C. 2007. Patch size and isolation effects on epiphytic and epiphyllous bryophytes in the fragmented Brazilian Atlantic forest. Biological Conservation 134: 415-427.
Alvarenga, L.D.P., Pôrto, K.C. \& Oliveira, J.R.P.M. 2010. Habitat loss effects on spatial distribution of non-vascular epiphytes in a Brazilian Atlantic forest. Biodiversity Conservation 19: 619-635.

Alvarenga, L.D.P., Pôrto, K.C. \& Silva, M.P.P. 2009. Relations between regional-local habitat loss and metapopulation properties of epiphyllous bryophytes in the Brazilian Atlantic forest. Biotropica 41(6): 682-691.

Amorin E.T. 2013. Estudo florístico e ecológico das briófitas da Serra Negra (Minas Gerais) e sua relação com outras áreas do sudeste do Brasil. Dissertação de Mestrado, Universidade Federal de Juiz de Fora, Juiz de Fora.

Amorim, E.T., Carvalho, F.A., Santos, N.D. \& LuiziPonzo, A. 2017. Distribution of Bryophytes in SouthEastern Brazil: An Approach on Floristic Similarity and Environmental Filtering. Cryptogamie Bryologie, 38: 3-17.

Bates, J.W. 1992. Influence of chemical and physical factors on Quercus and Fraxinus epiphytes at Loch Sunart, western Scotland: a multivariate analysis. Journal of Ecology 80: 163-179.

Batista, W.V.S.M. \& Santos N.D. 2016. Can regional and local filters explain epiphytic bryophyte distributions in the Atlantic Forest of southeastern Brazil? Acta Botânica Brasílica 30: 462-472.

Batista, W.V.S.M., Pôrto, K.C. \& Santos, N.D. 2018. Distribution, ecology, and reproduction of bryophytes in a humid enclave in the semiarid region of northeastern Brazil. Acta Botânica Brasílica 32(2): 303-313.

Benites, V.M, Schaefer, C.E.G.R., Mendonça, E.S. \& Martin, N.L. 2001. Caracterização da matéria orgânica e micromorfologia de solos sob Campos de Altitude no Parque Estadual da Serra do Brigadeiro (MG). Revista Brasileira de Ciência do Solo 25: 661- 674.

Bordin, J. \& Yano, O. 2013. Fissidentaceae (Bryophyta) do Brasil. Boletim do Instituto de Botânica 22: 1-72.

Caiafa, A. \& Silva, A. 2005. Composição florística e espectro biológico de um campo de altitude no Parque Estadual da Serra do Brigadeiro, Minas Gerais - Brasil. Rodriguésia 56: 163-173.

Carmo, D.M. \& Peralta, D.F. 2016. Survey of bryophytes in Serra da Canastra National Park, Minas Gerais, Brazil. Acta Botânica Brasílica 30: 254-265.

Costa, D.P. 2009. Briófitas. In: J.R. Stehmann, R.C. Forzza, A. Salino, M, Cabral, D.P. Costa \& L.H.Y. Kamino (eds.). Plantas da Floresta Atlântica. Jardim Botânico do Rio de Janeiro, Rio de Janeiro, pp. 13-17.

Castro, T.A., Leoni, L.S. \& Rocha, M.J. 2011. Briófitas do Herbário "Guido Pabst"- GFJP, Carangola - MG. In: XIII Encontro Latino Americano de Iniciação Científica e IX Encontro Latino Americano de Pós-Graduação Universidade do Vale do Paraíba, pp. 1-6. 
Costa, D.P. 1999. Epiphytic bryophyte diversity in primary and secondary lowland rainforest in southeastern Brazil. Bryologist 102: 320-326.

Costa, D.P. \& Peralta, D.F. 2015. Bryophytes diversity in Brazil. Rodriguésia 66: 1063-1071.

Costa, D.P., Santos, N.D., Rezende, M.A., Buck, W.R. \& Schäfer-Verwimp, A. 2015. Bryoflora of the Itatiaia National Park along an elevation gradient: diversity and conservation. Biodiversity and Conservation 24: 2199-2212.

Crum, H. 1994. Sphagnum leonii. A new species from Minas Gerais. Pabstia 5: 1-5.

Flora do Brasil 2020. 2020. Jardim Botânico do Rio de Janeiro. Available in http://floradobrasil.jbrj.gov.br/ (accessed on 15-VII-2020).

Frahm, J.P. 1991. Dicranaceae: Campylopodioideae, Paraleucobryoideae. Flora Neotropica, Monograph 54: 1-237.

Frahm, J.P. 2003. Manual of tropical bryology. Tropical Bryology 23: 1-196.

Germano, S.R. \& Pôrto K.C. 2006. Bryophyte communities in Atlantic forest remnant, state of Pernambuco, Brazil. Cryptogamie, Bryologie 27: 153-163.

Goffinet, B., Buck, W. \& Shaw, A. 2009. Morphology, anatomy and classification of the Bryophyta. In: B. Goffinet \& J. Shaw (eds.). Bryophyte Biology, 2 ed. Cambdrige University Press, Cambdrige, pp. 56-138.

Gradstein, S.R. 1997. The taxonomic diversity of epiphyllous bryophytes. Abstracta Botanica 21(1): 15-19.

Gradstein, S.R., Churchill, S.P. \& Salazar-Allen, N. 2001a. Guide to the bryophytes of tropical America. Memoirs of the New York Botanical Garden 1: 1-577.

Gradstein, S.R. \& Costa D.P. 2003. The Hepaticae and Anthocerotae of Brazil. Memoirs of the New York Botanical Garden 87: 1-318.

Gradstein, S.R., Griffin, III D., Morales, M. \& Nadkarni, N. 2001b. Diversity and habitat differentiation of mosses and liverworts in the cloud forest of Monteverde, Costa Rica. Caldasia 23: 203-212.

IBGE (Instituto Brasileiro de Geografia e Estatística). 2012. Manual técnico da vegetação brasileira. Rio de Janeiro.

Leoni, L.S. \& Tinte, V.A. 2004. Flora do Parque Estadual da Serra do Brigadeiro, Minas Gerais, Brasil: caracterização da vegetação e lista preliminar das espécies. Carangola, MG, Brasil: Faculdade de Filosofia, Ciências e Letras de Carangola, pp. 21-24.
Luizi-Ponzo, A., Siviero, T., Amorim, E., Henriques, D., Rocha, L., Gomes, H., Paiva, L., Rodrigues, R., Silva, I., Silva, A., Ribeiro, G., Gomes, C. \& Campeão, A. 2013. Briófitas do Parque Estadual do Ibitipoca no Herbário Prof. Leopoldo Kriegeer. In: R. Forzza, L. Neto, F.R. Salimena \& D. Zappi (Org.). Flora do Parque Estadual de Ibitipoca e seu entorno, 1ed Editora UFJF, Juiz de Fora, pp. 95-122.

Machado, P.S., Amorim, E.T., Siviero, T.S., Gomes, H.C.S. \& Luizi-Ponzo A.P. 2015. Novas ocorrências de espécies de Anthocerotophyta e Marchantiophyta para o estado de Minas Gerais, Brasil. Pesquisas, Botânica 67: 51-64.

Myers, N., Mittermeier, R.A., Mittermeier, C.G., Fonseca, G.A.B. \& Kent, J. 2000. Biodiversity hotspots for conservation priorities. Nature, 403: 853-858.

Newmaster, S.G., Belland, R.J., Arsenault, A., Vitt, D.H. \& Stephens, T.R. 2005. The ones we left behind: Comparing plot sampling and floristic habitat sampling for estimating bryophyte diversity: estimating bryophyte diversity. Diversity and Distributions 11: 57-72.

Oliveira-Silva, M.I.M.N., Mila, A.I. \& Yano, O. 2002. Aspectos ecológicos de briófitas em áreas preservadas de mata atlântica, Rio Janeiro, Brasil. Tropical Bryology 22: $77-102$.

Plano de Manejo PESB - Parque Estadual da Serra do Brigadeiro. 2007. Governo do Estado de Minas Gerais. Secretaria de Estado de Meio Ambiente e Desenvolvimento Sustentável - SEMAD, pp. 1-89.

Pócs, T. 1982. Tropical Forest Bryophytes. In: A.J.E., Smith (ed.). Bryophyte Ecology. Chapman and Hall, London, New York, pp. 59-103.

Puttick, M.N., Morris, J.L., Williams, Cox, C.J., Edwards D., Kenrick, P., Pressel, S., Wellman, C.H., T.A., Schneider, H., Pisani, D. \& Donoghue, D.P. 2018. The interrelationships of land Plants and the nature of the ancestral Embryophyte. Current Biology 28: 733-745.

Qiu, Y.L., Li, L., Wang, B., Chen, Z., Knoop, V., GrothMalonek, M., Dombrovska, O., Lee, J., Kent, L., Rest, J., Estabrook, G.F., Hendry, T.A., Taylor, D.W., Testa, CH.M., Ambros, M., Crandall-Stotler, B., Duff, R.J., Stech, M., Frey,W., Quandt, D. \& Davis, CH.C. 2006. The deepest divergences in land plants inferred from phylogenomic evidence. Proceedings of the National Academy of Sciences 103 (42): 15511-15516.

Rezende, C.L., Scarano, F.R., Assad, E.D., Joly, C.A., Metzger, J.P., Strassburg, B.B.N., Tabarelli, M., Fonseca, G.A. \& Mittermeier, R.A. 2018. From hotspot to hopespot: An opportunity for the Brazilian Atlantic Forest. Perspectives in Ecology and Conservation 16 (4): 208-214. 
Robbins, R.G. 1952. Bryophyte ecology of a dune area in New Zealand. Vegetation Acta Geobotanica 4: 1-31.

Santos, N.D. \& Costa, D.P. 2008. A importância de reservas particulares do patrimônio natural para a conservação da brioflora da Mata Atlântica: um estudo em El Nagual, Magé, RJ, Brasil. Acta Botânica Brasílica 22: 359-372.

Santos, E.D., Carmo, D. \& Peralta, D.F. 2017. Bryophytes of the cloud forest of Pico do Marumbi State Park, Paraná, Brazil. Check List 13: 959-986.

Sharp, A.J., Crum, H. \& Eckel, P. 1994. The Moss flora of Mexico. Memoirs of the New York Botanical Garden 69: 1-1113.

Siviero, T.S. \& Luizi-Ponzo, A.P. 2015. Briófitas de diferentes fitofisionomias florestais e campestres: estudo em uma área de conservação no sudeste do Brasil-Parque Estadual do Ibitipoca. Pesquisas, Botânica 67: 101-117.

Söderström, L., Hagborg, A., Konrat, M., BartholomewBegan, S., Bell, D., Briscoe, L., Brown, E., Cargill, D.C., Costa, D.P., Crandall-Stotler, B.J., Cooper, E., Dauphin, G., Engel, J., Feldberg, K., Glenny, D., Gradstein, S.R., He, X., Hentschel, J., IlkiuBorges, A.L., Katagiri, T., Konstantinova, N.A., Larraín, J., Long, D., Nebel, M., Pócs, T., Puche, F., Reiner-Drehwald, E., Renner, M., Sass-Gyarmati, A., Schäfer-Verwimp, A., Segarra-Moragues, J., Stotler, R.E., Sukkharak, P., Thiers, B., Uribe, J., Váňa, J., Wigginton, M., Zhang, L. \& Zhu, R.L. 2016. World checklist of hornworts and liverworts. PhytoKeys 59: 1-828.
Sousa, R.V. \& Câmara, P.E.A.S. 2015. Survey of the bryophytes of a gallery forest in the National Park of Serra do Cipó, Minas Gerais, Brazil. Acta Botânica Brasílica 29: 24-29.

Stehmann, J.R., Forzza, R.C., Salino, A., Sobral, M., Costa, D.P. \& Kamino, L. 2009. Plantas da Floresta Atlântica. Jardim Botânico do Rio de Janeiro, Rio de Janeiro, pp. 3-501.

Thiers, B. 2020. Index Herbariorum: A Global Directory of Public Herbaria and Associated Staff. New York Botanical Garden's Virtual Herbarium (http://sweetgum. nybg.org/science/ih/)

Vanderpoorter, A. \& Goffinet, B. 2009. Introduction to Bryophytes. Cambridge University Press, New York, pp. 6-329.

Yano, O. \& Carvalho, A. 1995. Briófitas da Serra da Piedade, Minas Gerais, Brasil. In: Anais do IX Congresso da Sociedade Botânica de São Paulo, pp. $15-25$.

Yano, O. \& Luizi-Ponzo, A.P. 2014. Adições à Brioflora do Parque Estadual do Ibitipoca, Minas Gerais, Brasil. Revista de Biologia Neotropical 11(2): 71-96.

Yano, O. \& Peralta, D.F. 2011a. Flora da Serra do Cipó, Minas Gerais: Briófitas (Anthocerotophyta, Bryophyta e Marchantiophyta). Boletim de Botânica da Universidade de São Paulo 29: 135-211.

Yano, O. \& Peralta, D.F. 2011b. Bryophytes from Serra de São José, Tiradentes, Minas Gerais, Brasil. Boletim de Botânica da Universidade de São Paulo 21: 141-172. 